ANSA/CR-CY) 206150

$$
\begin{aligned}
& \text { FWAL } \\
& \text { N-SI-CW } \\
& \text { OCIT } \\
& \text { OSTLO }
\end{aligned}
$$

\title{
A method for preparation, storage and activation of large populations of immotile sea urchin sperm
}

Gerácimo E. Bracho, Jennifer J. Fritch and Joseph S. Tash

Department of Molecular \& Integrative Physiology, University of Kansas Medical Center, 3901 Rainbow Boulevard, Kansas City, Kansas 66160-7401

Running Title: Preparation of immotile sperm

Submitted: BBRC 7/2/97

Accepted Date: 7/7/97 
ABSTRACT Reversible protein phosphorylation is associated with initiation and modulation of sperm flagellar motility. Many studies aimed at examining the signal transduction mechanisms underlying the expression of motility have relied on detergent-permeabilized sperm reactivated with exogenous ${ }^{32} \mathrm{P}$-ATP. However, the reactivation conditions allow variable levels of motility to be expressed and phosphorylation of many proteins that appear to be unrelated to sperm motility. Thus, identification of the few relevant proteins is difficult. We have developed a method to collect and keep sperm immotile until reactivated for analysis to normal motility levels. Artificial sea water (ASW) buffered with $5 \mathrm{mM} 2-[\mathrm{N}$-morpholino] $]$ thanesulfonic acid at $\mathrm{pH} 6.0$ and containing $50 \mathrm{mM} \mathrm{KCl}$, allows collection and storage of immotile sea urchin sperm for up to $96 \mathrm{~h}$ at $4-5^{\circ} \mathrm{C}$. Motility under these conditions is essentially zero, but sperm is rapidly reactivated to normal motility by diluting with $\mathrm{ASW}$ to standard $\mathrm{pH}(8.0)$ and $\mathrm{KCl}$ concentration $(10 \mathrm{mM})$ 
${ }^{a}$ KEY WORDS: MES, 2-[N-morpholino]ethanesulfonic acid; TAPS, Ntris[hydroxymethyl]-3-aminopropanesulfonic acid; STS, shuttle transportation system. 
Research in several laboratories supports the idea that reversible protein phosphorylation is associated with initiation and modulation of sperm motility (1-5). Many studies aimed at examining the intracellular signaling pathways that initiate the activation of flagellar motility have relied on detergent-permeabilized sperm reactivated with exogenous ${ }^{32}$ P-ATP $(2,6)$. However, the normal conditions of sperm collection and reactivation allow variable levels of motility to be expressed prior to analysis (7), as well as phosphorylation of many sperm proteins that are not clearly related to flagellar motility $(1,8)$. Thus, identification of the few relevant proteins that are rapidly phosphorylated during the initial stages of activation is difficult. This may be due to the destruction of compartmentalization boundaries inherent to the permeabilization process. Metabolic inhibitors or extended incubations at cold temperatures prior to reactivation of motility have been used to try to reverse the effects of background motility $(3,9,10)$. However, these methods depend on endogenous enzyme activities that may reverse the signals originally induced in the background motility $(3,11-13)$. A reliable method of collecting large amounts of immotile sperm capable of normal activation when required would solve this problem.

Our laboratory has recently been investigating the effect of microgravity on the signal transduction mechanisms of sperm motility activation. Previous studies have shown that sperm have greater velocity and amplitude of flagellar bending in microgravity (14). Signal transduction experiments in microgravity require that samples be prepared, loaded and stored in flight hardware not less than 18 hours prior to the scheduled launch of the Space Shuttle. In addition, following launch there is a time lapse of approximately $20 \mathrm{~h}$ that is necessary before the shuttle crew can begin microgravity experiments. These circumstances require that sperm samples be 
prepared in a buffer that keeps them immotile from several hours to a few days, as well as allows them to be activated to normal motility when required.

The method described in this paper is a modification of earlier methodologies used to study activation of respiration and motility of sea urchin sperm (15). The present method makes possible the preparation of large quantities of quiescent sea urchin sperm that can be stored for several days at $4-5^{\circ} \mathrm{C}$ without the use of exogenous metabolic inhibitors or detergents. There is no indication of adverse effects of buffer or storage conditions on the motile properties of the activated sperm. This method presents a potential solution to the high background and nonspecific protein phosphorylation of detergent-permeabilized sperm reactivation experiments. 


\section{MATERIALS AND METHODS}

Buffers. The following buffers were routinely used: artificial sea water(ASW) $\mathrm{pH} 8.0$, hardware sea water (HSW) pH 8.3, sperm storage buffer (SSB) pH 6.0 and MES ${ }^{\text {a }}$ sperm storage buffer (MSSB) pH 6.0. The chemical composition of these buffers is presented in Table 1. Adjustment of HSW to $\mathrm{pH} 8.3$ is to make the final solution $\mathrm{pH} 8.0$ after addition of sperm in SSB or MSSB. In addition, the following buffers were used: motility assay buffer (MAB), contained $1 \mathrm{mg} / \mathrm{ml}$ BSA in ASW; and non-motility assay buffer (NMAB), had $1 \mathrm{mg} / \mathrm{ml} \mathrm{BSA} \mathrm{in} \mathrm{MSSB} \mathrm{or} \mathrm{SSB.}$ Addition of BSA to these buffers prevents sperm from sticking to the plastic surfaces used in motility measurements.

Preparation of Sperm. Ripe sea urchins (Stronglyocentrotus purpuratus and Lytechinus pictus) were obtained from Marinus (Long Beach, CA). For most of the experiments described in this paper, animals were washed by sequential immersion into 3 trays containing $2 \mathrm{~L}$ each of cold SSB $\left(\sim 5^{\circ} \mathrm{C}\right)$ to remove sea water. Animals were bathed in each tray for $30-45 \mathrm{sec}$ using latex rubber gloves and then placed briefly on paper towels to drain excess buffer. Shedding of gametes was induced by intracoelomic injection of $1-2 \mathrm{ml}$ cold $0.5 \mathrm{M} \mathrm{KCl}$ through several sites on the peristomial membrane of the oral side. Sperm were collected by inverting male sea urchins over beakers filled with cold SSB or MSSB. Sperm were allowed to settle for 30-40 min at $5{ }^{\circ} \mathrm{C}$ and then carefully collected with a Pasteur pipette, to avoid resuspension and excessive dilution with buffer. A pool of sperm from several animals was made to minimize individual variations. A few experiments were carried out with sperm from animals that were not washed 
with SSB prior to spawning. In these instances, sperm were also collected in SSB or MSSB as described above. In addition, a portion of sperm was collected directly into ASW for use as a control to assess the activation of motility of sperm collected in SSB or MSSB. Storage of sperm was always done at $4-5^{\circ} \mathrm{C}$.

Activation and Quantitation of Sperm Motility. Immotile sperm were activated by dilution into activation buffers (ASW or HSW) as described below. Control experiments were carried out by transferring sperm into non activation buffers (SSB or MSSB). Quantitative analysis of sperm motility was performed as previously described $(3,12,16,17)$. Adjustments were made to compensate for the greater average curvilinear velocity of sea urchin sperm compared to other species. Motility chambers were prepared by attaching a press-on letter ' $O$ ' (Chartpak RDC 49) to the center of a plastic petri dish (Falcon 1029). The $32 \mu \mathrm{m}$ thick letters created a well of fixed depth. Sperm were diluted into HSW for activation, or SSB or MSSB for control. The sperm suspension was then immediately diluted into MAB or NMAB. Dilutions were made at a ratio of $1 \mu \mathrm{l}$ of sperm suspension to $200 \mu \mathrm{l}$ of MAB or NMAB, to yield approximately $10-30$ sperm per field during video microscopy. A $10 \mu \mathrm{l}$ aliquot of the diluted sperm was then placed in the center of the well. A plastic cover slip (Fisher 12-547) was then carefully pressed over the suspension using the reverse end of a $5 \mathrm{ml}$ Pipetman pipette tip (Fisher 21-375-3) to seal the chamber and ensure uniform well depth and distribution of sperm. Motility was quantitated within 2 min of sperm dilution. To examine the effect of temperature on motility activation, some experiments were performed with sperm that were allowed to incubate at room temperature in SSB or MSSB prior to initial dilution into SSB or MSSB and subsequent dilution into NMAB for motility 
analysis. Times of incubation are described where appropriate.

Video microscopy was performed at room temperature $\left(22^{\circ} \mathrm{C}\right)$ using a Nikon Diaphot inverted microscope with 20X BM phase objective and $1 \mathrm{X}$ video adapter connected to a Dage CCD72 camera. The video signal was sent via a video time clock (For.A VTG33) to a Panasonic VHS video recorder (Model AG-2550). Motility was recorded on multiple viewing fields for 15 sec per field for a total of 4 minutes.

Video tapes were analyzed using the CellTrack/s, version 5.0 (Motion Analysis Corp.). Adjustable parameters were set as follows: frame rate $=60 / \mathrm{sec}$; duration of capture $=23$ frames; minimum motility $=25 \mu \mathrm{m} / \mathrm{sec} ;$ maximum burst speed $=700 \mu \mathrm{m} / \mathrm{sec} ;$ micron $/$ pixel ratio $=$ $1.2401 ;$ minimum cell size $=3$ pixels; maximum cell size $=12$ pixels; number of cells to find per well $=400 ;$ minimum number of fields per sample $=3$. Data from a minimum of 400 cells was collected. Replicate wells were analyzed for each treatment. Parameters quantitated were percent motility (MOT), curvilinear velocity (VCL), straight line velocity (VSL), linearity (LIN), amplitude of lateral head displacement (ALH), average path velocity (VAP), and population progressiveness $(\mathrm{PRG}=\mathrm{MOT} \times \mathrm{VCL} / 100$ ). For simplicity, we only discuss in this paper percent motility, curvilinear velocity and population progressiveness. Statistics were calculated from replicate well data.

\section{RESULTS AND DISCUSSION}

The present study was initiated with the purpose of finding conditions in which sea urchin sperm could be collected and stored completely immotile to allow sufficient time to perform 
microgravity experiments. These conditions would enable immotile sperm to be activated to normal levels of motility when required, and ideally, this activation would elicit only signaling pathways related to the initiation of flagellar motility. This latter case would facilitate detection of small differences in phosphorylation between initiation of motility in microgravity compared to normal gravity. Finally, these conditions would be relatively mild, to prevent any adverse effect on the motile properties of the sperm. Previous methods of collection of immotile sperm allow spawning directly on the surface of the animal to avoid contact of the dry sperm with the activation conditions of ASW. In our method, animals are washed in SSB prior to spawning to minimize the risk of sperm contact with the $\mathrm{pH}$ and potassium conditions which promote motility activation. In addition, this method yields immotile sperm that do not have to be treated with metabolic inhibitors to reduce background motility $(9,18)$.

The chemical composition of buffers used for collection, storage and activation of immotile sperm is shown in Table 1. The buffer composition was based on earlier work by Christen et al. (15), which demonstrated that high potassium concentration and low $\mathrm{pH}$ inhibited sea urchin sperm motility. Results presented in this work indicate that the buffering capacity of solutions used for collection and storage determine the propensity of sperm to initiate motility activation. This is supported by the results shown in Figure 1, which indicate that SSB appeared to have enough buffering capacity to prevent sperm activation for the first $24 \mathrm{~h}$, but was insufficient after $48 \mathrm{~h}$ storage at $4-5^{\circ} \mathrm{C}$. Figure 1 also shows that activation of motility within SSB was enhanced by incubation at room temperature. Sperm activation was significantly higher after $72 \mathrm{~h}$ storage and the effect of incubation at room temperature was also significantly higher. In contrast, no activation was observed in sperm stored in MSSB for $48 \mathrm{~h}$ at $4-5{ }^{\circ} \mathrm{C}$ and incubated 
at room temperature for up to $60 \mathrm{~min}$ (Fig. 1). After $72 \mathrm{~h}$ storage at $4-5^{\circ} \mathrm{C}$, some activation of MSSB sperm was observed but only on sperm incubated for 30 and $60 \mathrm{~min}$ at room temperature prior to motility analysis. After $96 \mathrm{~h}$ storage at $4-5^{\circ} \mathrm{C}$ and $5 \mathrm{~min}$ incubation at room temperature, sperm stored in SSB showed motility activation that was significantly higher than the motility activation of sperm stored in MSSB. However, activation of sperm motility was similar in both buffers after 30 and $60 \mathrm{~min}$ room temperature incubation. Results presented in Fig. 1 could be attributed to the buffer composition of ASW, HSW, SSB and MSSB. Sperm activating buffers (ASW and HSW) had good buffering capacity at $\mathrm{pH} 8.0$ and $\mathrm{pH} 8.3$, due to the presence of $5 \mathrm{mM}$ TAPS (pKa $\sim 8.4$ at $25^{\circ} \mathrm{C}$ ). However, when the $\mathrm{pH}$ was adjusted to 6.0 (to make SSB), the buffers lost virtually all of their buffering capacity. It appears that the lack of buffering capacity of SSB at $\mathrm{pH} 6.0$, was sufficient to allow the sperm activation observed after $48 \mathrm{~h}$ storage at $4-5{ }^{\circ} \mathrm{C}$. In contrast, there was good buffering capacity in MSSB at $\mathrm{pH} 6.0$ with the addition of $5 \mathrm{mM}$ MES ( $\mathrm{pKa} \sim 6.1$ at $25^{\circ} \mathrm{C}$ ) to SSB, and sperm stored in MSSB remained immotile after $48 \mathrm{~h}$, and essentially remained immotile after $96 \mathrm{~h}$ storage at $4-5^{\circ} \mathrm{C}$. No significant differences were found in the motile properties of sperm stored for $96 \mathrm{~h}$ at $4-5^{\circ} \mathrm{C}$ in SSB or MSSB after activation by dilution in HSW and MAB, as shown in Fig. 2. It appears, however, that the motility parameters of sperm decreased substantially after $72 \mathrm{~h}$ storage in both buffers. The effect of these storage conditions on the functional properties of sperm, namely fertility, is under investigation. We found that increasing the MES concentration in MSSB to $10 \mathrm{mM}$ appears to be toxic to sperm. The activation of immotile sperm stored in MSSB containing $10 \mathrm{mM} \mathrm{MES,} \mathrm{was} \mathrm{significantly}$ lower when diluted in HSW than similar dilution of sperm stored in SSB or MSSB containing 5 mM MES (data not shown). We found no difference in the activation of sperm collected from 
animals that were washed with SSB or MSSB prior to spawning.

We did not attempt to determine whether there was a $\mathrm{pH}$ change in SSB due to presence of sperm, however, we speculate that some of the observed activation of sperm in SSB was due in part to a slight increase in $\mathrm{pH}$ during storage. Moreover, we observed a significant difference in the $\mathrm{pH}$ of SSB and MSSB at low temperature in the absence of sperm. After initial adjustment to $\mathrm{pH} 6.0$ at room temperature, SSB showed an increase of $0.2 \mathrm{pH}$ units after incubation overnight at $5^{\circ} \mathrm{C}$, whereas MSSB showed no $\mathrm{pH}$ change. Finally, activation could also be due to exposure of sperm to sea water remaining on the surface of the animals during spawning. This is consistent with the finding that fresh sperm from animals that were not washed with SSB prior to spawning, exhibited flagellar motility regardless of the buffer used for collection. These results were observed in sperm analyzed less than $2 \mathrm{~h}$ after collection and are summarized in Table 2. We found that sperm from these animals showed no significant activation $(<0.5 \%$ motility $)$ in either buffer when analyzed with no incubation at room temperature. However, after 5 min incubation at room temperature, both sperm exhibited activation of motility (percent motility was $5 \pm 1.4$ in SSB and $2 \pm 0.7$ in MSSB, Table 2). Activation of motility was considerably higher if incubation at room temperature was extended to $50 \mathrm{~min}$, particularly in sperm collected in SSB $(17 \pm 2.9$ percent motility in SSB versus $9 \pm 1.3$ in MSSB). These results also support the idea that the buffering capacity at $\mathrm{pH} 6.0$ provided by $5 \mathrm{mM}$ MES plays a key role in the inhibition of motility activation of sperm stored collected and stored in MSSB. Inhibition of motility by MSSB appears to be due to the effect of its chemical composition on the internal sperm $\mathrm{pH}$ and its high potassium concentration, which is similar to that found in echinoderm testicular luminal fluid (19-21). These inhibition conditions are reversed by dilution of sperm into HSW, thus allowing 
excellent activation.

In conclusion, we have formulated a chemically defined solution that allows preparation, storage and activation of immotile sea urchin sperm for up to 4 days at $4-5^{\circ} \mathrm{C}$ with no apparent adverse effects on the motile properties of the activated sperm. This method was used to prepare immotile sperm for use in microgravity experiments performed on January and May 1997 Space Shuttle flights (STS-81 and STS-84). In addition, we have used this method in our laboratory for long term incubation and activation experiments with sea urchin sperm. Data from these experiments indicate a low background of phosphorylation and that only a few proteins show changes in the degree of phosphorylation during the initial stages of flagellar motility activation (paper in preparation). This is in contrast with similar experiments using detergentpermeabilized sperm in which numerous proteins appear to undergo phosphorylation changes during the reactivation process in a high background of phosphorylation $(1,3,8,12,22)$. 


\section{ACKNOWLEDGEMENTS}

Supported by grant NAG-2-1016 from NASA and grant HD-33994 from NIH to the

Center for Reproductive Sciences. 


\section{REFERENCES}

1. Chaudhry, P.S., Creagh, S., Yu, N., and Brokaw, C.J. (1995) Cell Motil. Cytoskeleton 32, 6579.

2. Bracho, G.E. and Tash, J.S. (1995) Methods Cell Biol. 47, 447-458.

3. Ahmad, K., Bracho, G.E., Wolf, D.P., and Tash, J.S. (1995) Arch. Androl. 35, 187-208.

4. Leclerc, P., de Lamirande, E., and Gagnon, C. (1996) Biol. Reprod. 55, 684-692.

5. Si, Y. and Okuno, M. (1995) Biol. Reprod. 53, 1081-1087.

6. Brokaw, C.J. (1987) J. Cell. Biochem. 35, 175-184.

7. Brokaw, C.J. (1984) Ann. N. Y. Acad. Sci. 438, 132-141.

8. Tash, J.S. and Means, A.R. (1983) Biol. Reprod. 28, 75-104.

9. Lindemann, C.B. (1978) Cell. 13, 9-18.

10. Tash, J.S., Hidaka, H., and Means, A.R. (1986) J. Cell Biol. 103, 649-655.

11. Takahashi, D., Murofushi, H., Ishiguro, K., Ikeda, J., and Sakai, H. (1985) Cell Struct. Funct. 10, 327-337.

12. Tash, J.S., Krinks, M., Patel, J., Means, R.L., Klee, C.B., and Means, A.R. (1988) J. Cell Biol. 106, 1625-1633.

13. Vijayaraghavan, S., Stephens, D.T., Trautman, K., Smith, G.D., Khatra, B., da Cruz, e.F., and Greengard, P. (1996) Biol. Reprod. 54, 709-718.

14. Engelmann, U., Krassnigg, F., and Schill, W.B. (1992) J. Androl. 13, 433-436.

15. Christen, R., Schackmann, R.W., and Shapiro, B.M. (1986) J. Physiol. (Lond. ) 379, 347365.

16. Mujica, A., Neri-Bazan, L., Tash, J.S., and Uribe, S. (1994) Mol. Reprod. Dev. 38, 285-292.

17. Tash, J.S. and Wolf, D.P. (1989) in Perspectives in andrology (M. Serio, Ed.), pp. 407-419. Sereno Symposia Publications from Raven Press, New York.

18. Rikmenspoel, R., Jacklet, A.C., Orris, S.E., and Lindemann, C.B. (1973) J. Mechanochem. Cell Motil. 2, 7-24. 
19. Lee, H.C., Johnson, C., and Epel, D. (1983) Dev. Biol. 95, 31-45.

20. Christen, R., Schackmann, R.W., and Shapiro, B.M. (1982) J. Biol. Chem. 257, 14881 14890.

21. Rothschild, Lord (1948) J. Exp. Biol. 25, 344-352.

22. Dey, C.S. and Brokaw, C.J. (1991) J. Cell Sci. 100, 815-824. 


\section{FIGURE LEGENDS}

FIG. 1. Effect of spawning pretreatment, storage and incubation conditions on sperm motility. Animals were washed in SSB and spawned in SSB or MSSB as described in Materials and Methods. Sperm stored in SSB and MSSB at $4-5^{\circ} \mathrm{C}$ were incubated at $22{ }^{\circ} \mathrm{C}$ for 5,30 and 60 min prior to an initial dilution in SSB and MSSB and a final dilution in NMAB where motility was quantitated. Bars indicate mean value \pm standard deviation. Missing bars indicate $0 \%$ motility for all replicates. Motility parameters are defined in Materials and Methods. Statistical comparisons of motility between SSB and MSSB at each 5, 30 and 60 min time point were all significantly different $(\mathrm{P}<0.02$ or better) except for 30 and $60 \mathrm{~min}$ at $96 \mathrm{hr}$ (no significant difference indicated by $*$ ).

FIG. 2. Effect of storage conditions on sperm motile properties. Animals were washed and spawned in SSB or MSSB as described in Materials and Methods. Sperm stored in SSB and MSSB at $4-5^{\circ} \mathrm{C}$ were activated at $22^{\circ} \mathrm{C}$ by an initial dilution in $\mathrm{HSW}$ followed by a final dilution in $\mathrm{MAB}$ where motility was quantitated. Bars indicate mean value \pm standard deviation. Motility parameters are defined in Materials and Methods. No significant differences were found in any of the motile properties of activated sperm stored in SSB and MSSB. 
TABLE 1

Composition of Buffers for Collection, Storage and Activation of Immotile Sperm

\begin{tabular}{lcccc}
\hline & ASW & HSW & SSB & MSSB \\
\hline $\mathrm{pH}$ & 8.0 & 8.3 & 6.0 & 6.0 \\
$\mathrm{KCl}$ & 10 & 10 & 50 & 50 \\
$\mathrm{MES}$ & - & - & - & 5 \\
$\mathrm{TAPS}$ & 5 & 5 & 5 & 5 \\
$\mathrm{NaCl}$ & 425 & 425 & 425 & 425 \\
$\mathrm{MgCl}_{2}$ & 27 & 27 & 27 & 27 \\
$\mathrm{MgSO}_{4}$ & 29 & 29 & 29 & 29 \\
$\mathrm{CaCl}_{2}$ & 10 & 10 & 10 & 10 \\
$\mathrm{NaHCO}_{3}$ & 2.4 & 2.4 & 2.4 & 2.4 \\
\hline
\end{tabular}

Note. All concentrations are given in mM. The symbol " - " means no MES addition. 
TABLE 2

Motility Parameters of Sperm from Animals Not Washed Prior to Spawning

\begin{tabular}{lcc}
\hline & \multicolumn{3}{c}{ Incubation time (min) } \\
Parameter & 5 & 50 \\
\hline SSB MOT $(\%)$ & $5 \pm 1.4$ & $17 \pm 2.9$ \\
MSSB MOT $(\%)$ & $2 \pm 0.7$ & $9 \pm 1.3$ \\
SSB VCL $(\mathrm{nm} / \mathrm{sec})$ & $111 \pm 7.8$ & $282 \pm 23.5$ \\
MSSB VCL $(\mathrm{nm} / \mathrm{sec})$ & $220 \pm 15.4$ & $162 \pm 10.5$ \\
SSB PRG $(\mathrm{nm} / \mathrm{sec})$ & $5.6 \pm 1.4$ & $48 \pm 5.6$ \\
MSSB PRG $(\mathrm{nm} / \mathrm{sec})$ & $4.4 \pm 1.3$ & $15 \pm 2.3$ \\
\hline
\end{tabular}

Note. Incubations were performed at room temperature $\left(22^{\circ} \mathrm{C}\right)$ prior to initial dilution of sperm into SSB or MSSB and subsequent dilution into NMAB for motility quantitation. Numbers indicate mean value \pm standard deviation. 


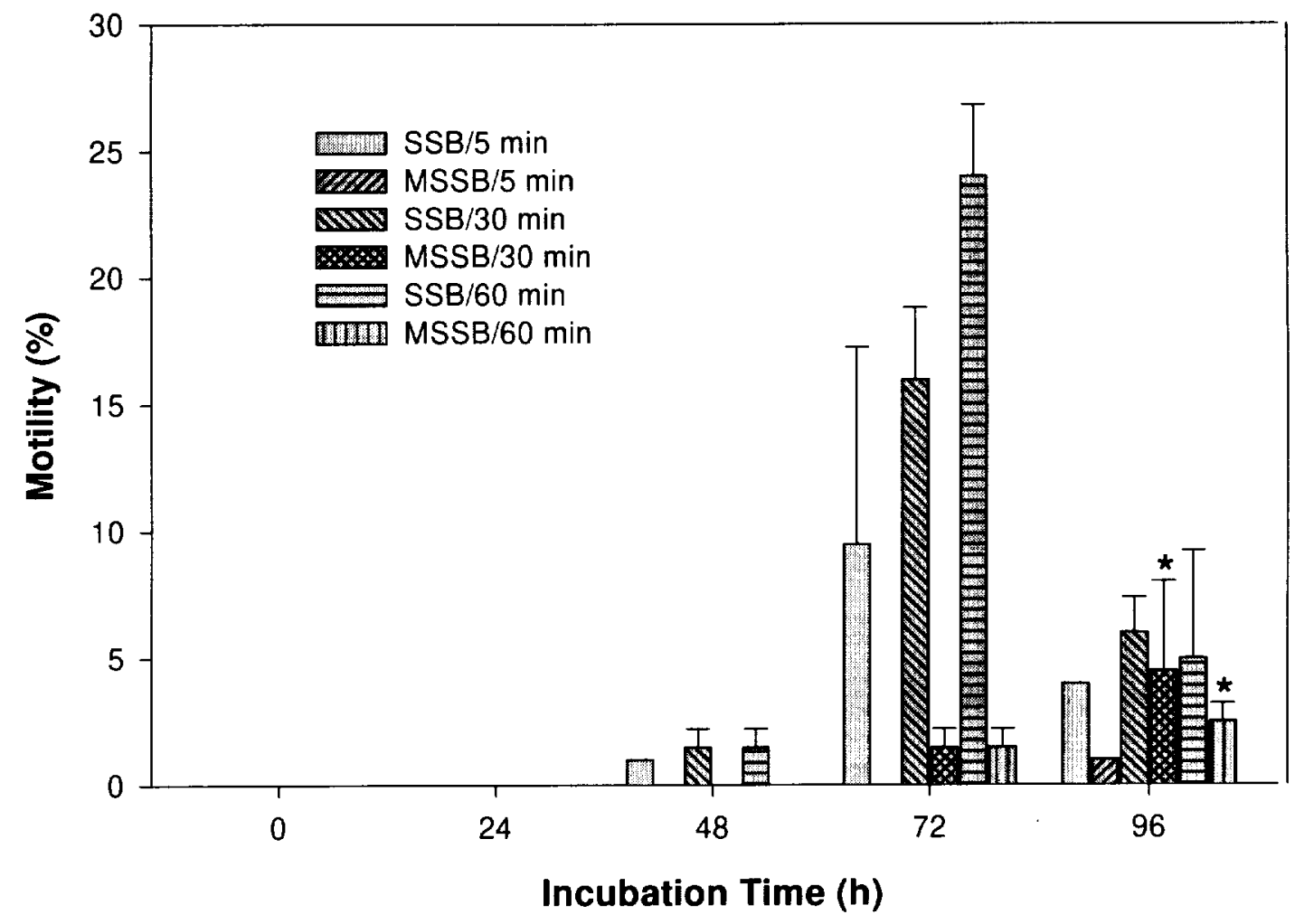




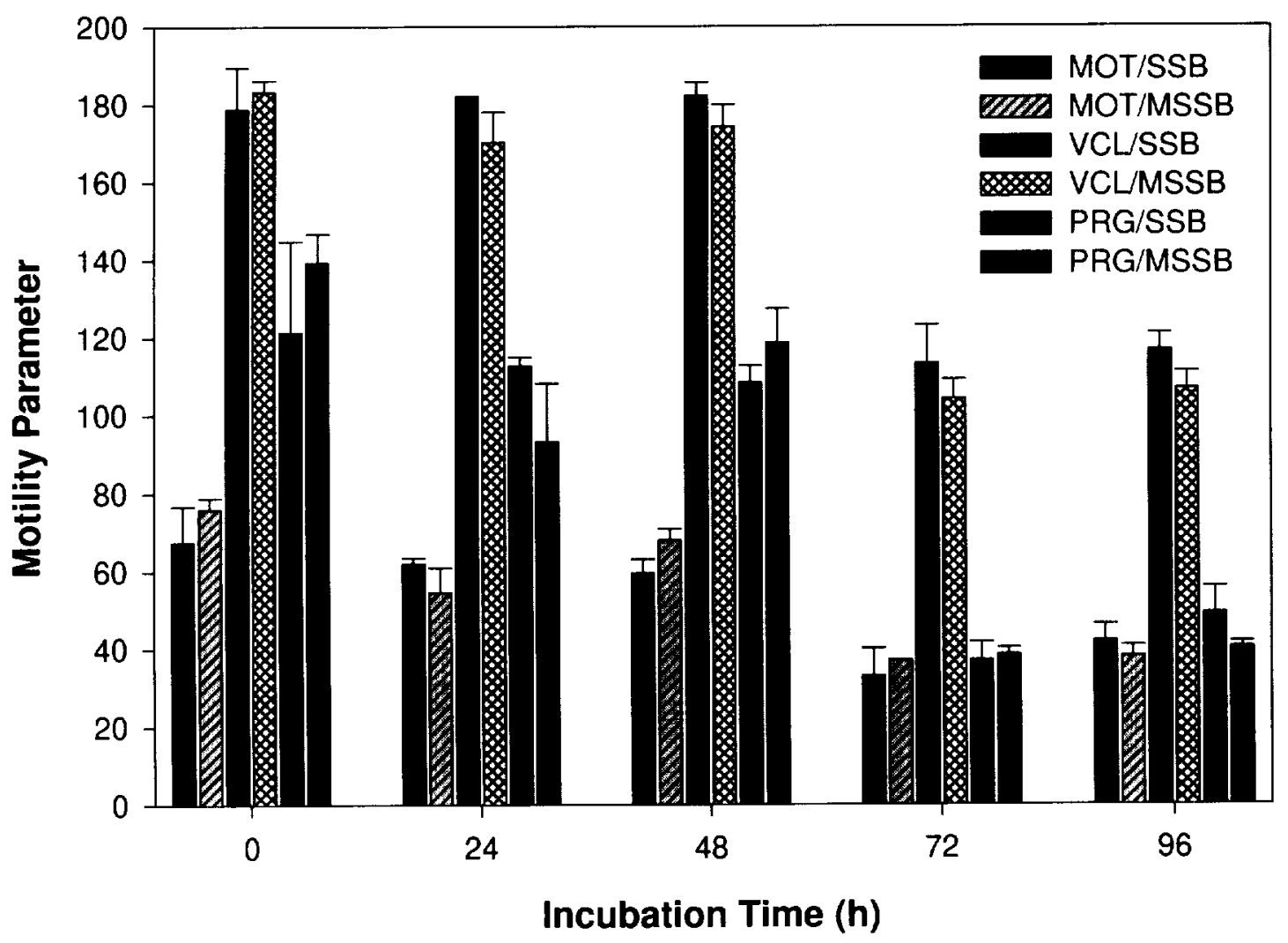

